\title{
High-speed germanium pin photodiodes integrated on silicon-on-insulator nanophotonic waveguides
}

\author{
D. Benedikovic ${ }^{1, *}$, L. Virot ${ }^{2}$, G. Aubin ${ }^{1}$, J.-M. Hartmann², B. Szelag², F. Amar ${ }^{1}$, X. Le Roux ${ }^{1}$, C. Alonso-Ramos ${ }^{1}$, P. \\ Crozat $^{1}$, D. Marris-Morini ${ }^{1}$, E. Cassan ${ }^{1}$, C. Baudot ${ }^{3}$, F. Boeuf ${ }^{3}$, J.-M. Fédéli ${ }^{2}$, C. Kopp ${ }^{2}$, and L. Vivien ${ }^{1}$ \\ ${ }^{1}$ Centre de Nanosciences et de Nanotechnologies, CNRS, Univ. Paris-Sud, Université Paris-Saclay, C2N - Palaiseau, 91120 \\ Palaiseau, France \\ ${ }^{2}$ University Grenoble Alpes and CEA, LETI, 38054 Grenoble, France \\ ${ }^{3}$ STMicroelectronics, Silicon Technology Development, 38926 Crolles, France \\ *daniel.benedikovic@c2n.upsaclay.fr
}

\begin{abstract}
Hetero-structured silicon-germanium-silicon photodetectors operating under low-reverse-voltages with high responsivity, fast response, and low dark-current levels are reported. A bit-error-rate of $10^{-9}$ is experimentally achieved for conventional data rates of 10, 20, and $25 \mathrm{Gbps}$, providing optical

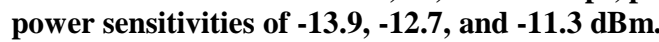

Keywords - silicon nanophotonics, germanium, silicon-oninsulator, optical photodetectors, complementary metal-oxidesemiconductor technology

\section{INTRODUCTION}

Integration of optical photodetectors on mature silicon-oninsulator (SOI) substrates is key for a variety of applications in nanophotonics ranging from sensing and health monitoring to object recognition and optical communications. Although silicon ( $\mathrm{Si}$ ) is at the heart of modern micro-electronics industry, its electronic bandgap, with an energy of $1.12 \mathrm{eV}$ and a cut-off wavelength of $\sim 1.1 \mu \mathrm{m}$, precludes the fabrication of performant detectors operating at near-infrared (near-IR) wavelengths, historically harnessed by fiber-optics communication systems [1].

Germanium (Ge), a group-IV elemental semiconductor as $\mathrm{Si}$, is regarded as a prime choice for efficient light detection in this $1.3-1.55 \mu \mathrm{m}$ spectral region. High optical absorption, good crystalline quality films, and cost-effective CMOScompatible process flows are the reasons why germanium is so promising for scalable group-IV optical interconnects [1, 2], as no complex heterogeneous integration is needed.

Different implementations of Ge pin photodetectors have been demonstrated over the last decade [3-12]. From a technological point of view, homo-junction photodetectors require both heavy dopings and metallic contacts formation directly on Ge. This results in a variety of shortcomings such as high losses, reduced responsivity, and large dark-currents [3-7]. Si-contacted pin detectors, with both vertical and lateral hetero-junctions, have been elaborated [8-12]. This scheme not only eases device fabrication, but also results in convincing performances. Experimentally, improved responsivity, faster operation, as well as lower dark-currents were achieved at telecom and datacom wavelengths.

Here, we report on high-bit-rate optical link operation of hetero-structured pin photodetectors under low supply biases.
A $10^{-9}$ error-free detection of bit streams up to $25 \mathrm{Gbps}$ is demonstrated, with a power sensitivity of $-11.3 \mathrm{dBm}$.

\section{DEVICE DESIGN AND FABRICATION}

A schematics of pin photodetector with lateral silicongermanium-silicon ( $\mathrm{Si}-\mathrm{Ge}-\mathrm{Si}$ ) heterojunctions can be found in Figure 1a. Devices were fabricated on silicon-on-insulator (SOI) substrates with $220 \mathrm{~nm}$ thick Si layers and $2 \mu \mathrm{m}$ thick Buried OXides. They facilitate operation of TE-like waveguide mode across the $C$-band wavelengths referenced at $1.55 \mu \mathrm{m}$. The $260-\mathrm{nm}$-thick intrinsic Ge region is sandwiched between doped $\mathrm{Si}$ slabs and situated within a deeply etched Si cavity (60 $\mathrm{nm}$ remaining thickness) at the end of a Si waveguide. The off-chip coupling is performed with surface grating couplers to launch light from an optical fiber into the chip, while on-chip coupling exploits efficient but-waveguide-coupled light injection between single-mode $\mathrm{Si}$ waveguides and Si-Ge-Si photodetectors [12].

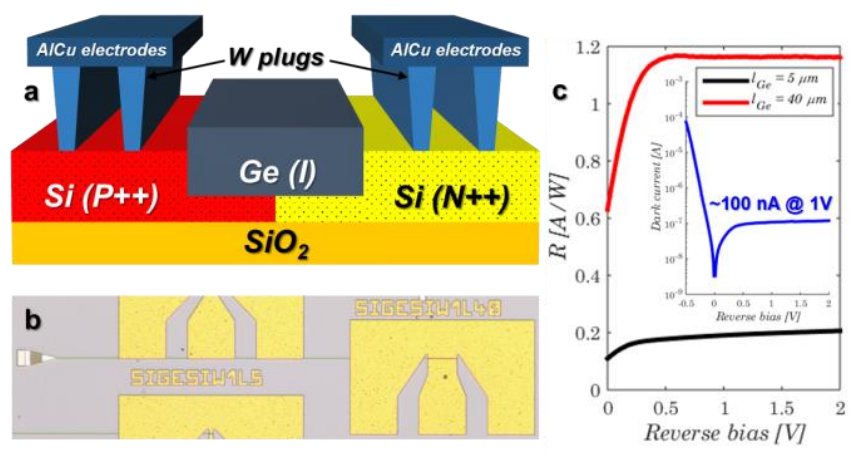

Fig. 1 (a) Schematics of a pin waveguide photodetector with a lateral Si-GeSi heterojunction. (b) Optical microscopy image of fabricated devices. (c) Responsivity as a function of reverse bias for $1 \mu \mathrm{m}$ wide pin photodetectors with two lengths. Inset: Dark current versus reverse voltage for a $1 \mu \mathrm{m}$ wide and $40 \mu \mathrm{m}$ long device.

Pin photodetectors were processed in CEA LETI's facilities using $200 \mathrm{~mm}$ SOI wafers and a CMOS production line. Fabrication details are provided in $[11,12]$. Figure $1 \mathrm{~b}$ shows an optical microscopy image of fabricated devices. The integration strategy used here merges but-waveguide coupling and lateral pin heterojunctions to have compact devices with tailored waveguide features. Such an integration simplifies fabrication, improves device responsivity and 
bandwidth. It is thus promising for the monolithic integration of a large range of passive and active nano-photonic devices with a reduced number of process steps, lowering production costs.

\section{DETECTOR PERFORMANCE}

Detectors were fully characterized by using static currentvoltage measurements, small-signal radio-frequency tests and large-signal data link acquisitions, as detailed in $[11,12]$.

Figure 1c shows the detector responsivity as a function of applied reverse bias. The power coupled to a detector was $11.2 \mathrm{dBm}$. Responsivities at $1 \mathrm{~V}$ bias are 0.19 and $1.19 \mathrm{~A} / \mathrm{W}$ for $5 \mu \mathrm{m}$ and $40 \mu \mathrm{m}$ long devices, respectively. The responsivity reaches a maximum at bias as low as $0.5 \mathrm{~V}$. After that, it remains virtually flat, with almost no voltage dependence. This trend was consistently observed on all measured devices. The Si-Ge-Si detectors carry the capability to sweep out the vast majority of photo-generated carriers within their lifetime. The inset of Fig. 1c shows the darkcurrent versus the reverse bias. Under $1 \mathrm{~V}$ reverse bias, the dark-current is $7 \mathrm{nA}$ for $5 \mu \mathrm{m}$ long devices and increases up to $100 \mathrm{nA}$ for $40 \mu \mathrm{m}$ long devices.
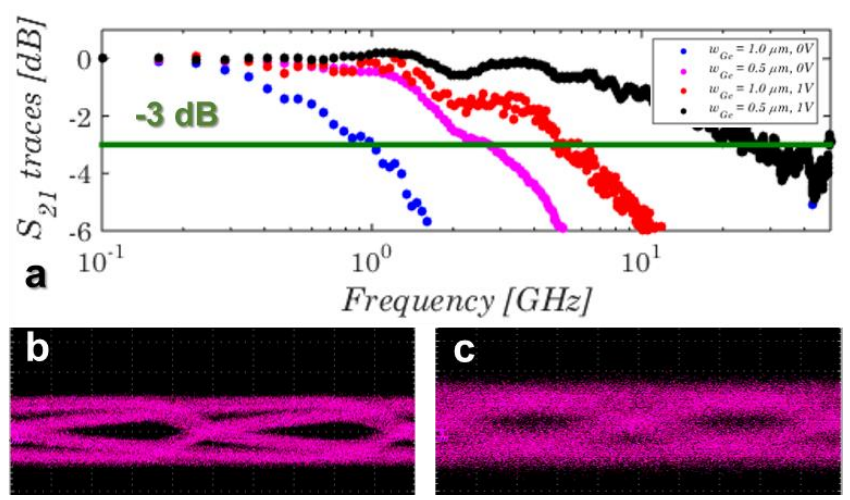

Fig. 2 (a) Normalized $S_{21}$ traces for various widths $40 \mu \mathrm{m}$ long pin photodetectors biased at $0 \mathrm{~V}$ and $1 \mathrm{~V}$. Measured eye diagrams under $1 \mathrm{~V}$ reverse bias: (b) $w_{\mathrm{Ge}}=1.0 \mu \mathrm{m}$ at $10 \mathrm{Gbps}$ and (c) $w_{\mathrm{Ge}}=0.5 \mu \mathrm{m}$ at $40 \mathrm{Gbps}$.

Figure 2a shows series of normalized $S_{21}$ frequency responses for various detector widths, as biased under $0 \mathrm{~V}$ and $1 \mathrm{~V}$ states. The device length was fixed to $40 \mu \mathrm{m}$. The $3 \mathrm{~dB}$ bandwidth was between 0.97 and $2.53 \mathrm{GHz}$ at $0 \mathrm{~V}$ for $1 \mu \mathrm{m}$ and $0.5 \mu \mathrm{m}$ widths detectors. The device cut-off frequency is restricted under $0 \mathrm{~V}$, because of the long transit time of carriers. Only the built-in electric field yields an efficient collection of photo-generated carriers. At $0 \mathrm{~V}$, the strength of that field is weak, however. Accordingly, eye diagrams remained closed in all cases. In opposition to that, increased bias significantly enhances the bandwidth, up to 5.1 and up to $25.3 \mathrm{GHz}$ for 1 $\mu \mathrm{m}$ and $0.5 \mu \mathrm{m}$ wide devices ( $1 \mathrm{~V}$ reverse bias). The cut-off frequency of detectors increases as the width of the intrinsic $\mathrm{Ge}$ region is reduced. As shown in Figs. 2b-c, under $1 \mathrm{~V}$ reverse bias, open eye diagrams were retrieved at $10 \mathrm{Gbps}$ for $1 \mu \mathrm{m}$ wide devices $40 \mathrm{~Gb}$ ps and for $0.5 \mu \mathrm{m}$ wide devices. Furthermore, we consistently observed that the measured cutoff frequency did not depend on the device length $[11,12]$. This indicates that the achievable bandwidth limit of hetero- structured Si-Ge-Si pin photodetector is not governed by RC delay.

Figure 3a shows bit-error-rate (BER) measurements as functions of the received optical power for a data rate of 10 Gbps. Devices were biased at $1 \mathrm{~V}$ and 3V. The optical power sensitivities, for a $10^{-9}$ error-less level were -10 and -13.9 $\mathrm{dBm}$, respectively. Furthermore, Fig. $3 b$ presents BER results at different data rates of 10,20 , and $25 \mathrm{Gbps}$. Power sensitivities of $-13.9,-12.7$, and $-11.3 \mathrm{dBm}$ are associated with these link rates, for a $10^{-9}$ BER threshold. Those BER determinations were performed on a $1 \mu \mathrm{m}$ wide by $40 \mu \mathrm{m}$ long pin photodetector.
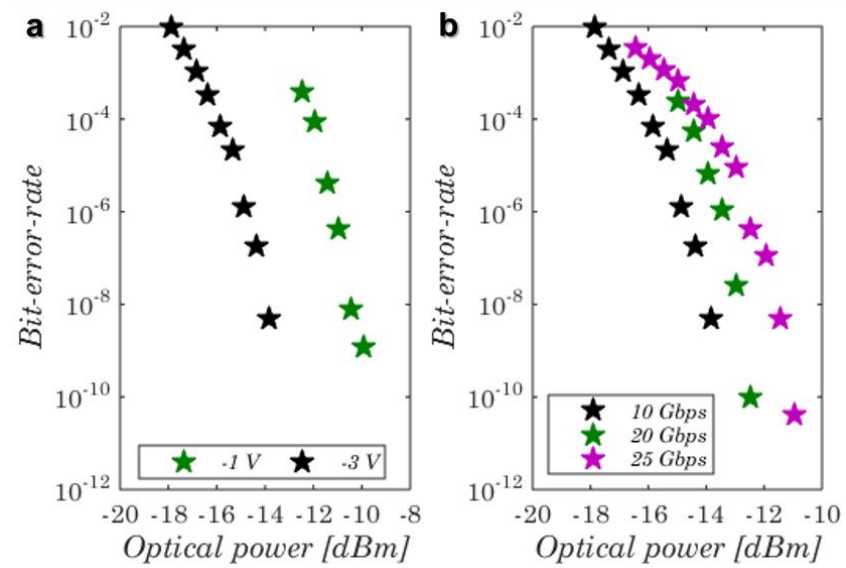

Fig. 3 Bit-error-rate measurements for a $1 \mu \mathrm{m}$ wide by $40 \mu \mathrm{m}$ long Si-Ge-Si pin photodetector functions of the input optical power, as performed (a) at $10 \mathrm{Gbps}$ data rate under low-reverse-biases of $1 \mathrm{~V}$ and $3 \mathrm{~V}$ and (b) at data rates of 10,20 , and $25 \mathrm{Gbps}$ under $3 \mathrm{~V}$ reverse voltage.

\section{CONCLUSION}

We demonstrated the superior high-speed performances of pin photodetectors with lateral Si-Ge-Si heterojunctions. Under low-bias operation, high responsivity, fast response, and low dark currents were simultaneously achieved.

\section{ACKNOWLEDGMENTS}

This work was supported by the European Research Council (ERC) under the European Union's Horizon 2020 research and innovation program (ERC POPSTAR - grant agreement No 647342).

\section{REFERENCES}

[1] L. Vivien and L. Pavesi, Handbook of Silicon Photonics (2013).

[2] J. Michel, et al., Nat. Photonics 4, 527-534 (2010).

[3] L. Vivien, et al., Opt. Express 17, 6252-6257 (2009).

[4] J. Joo, et al., Opt. Express 18, 16474-16479 (2010).

[5] S. Assefa, et al., Opt. Express 18, 4986-4999 (2010).

[6] L. Vivien, et al., Opt. Express 20, 1096-1101 (2012).

[7] H. Pan, et al., Opt. Express 20, 18145-18155 (2012).

[8] Y. Zhang, et al., Opt. Express 22, 11367-11375 (2014).

[9] H. T. Chen, et al., OSA J. Lightwave Technol. 33, 820-824 (2016).

[10] H. Chen, et al., Opt. Express 24, 4622-4631 (2016).

[11] L. Virot, et al., Opt. Express 25, 19487-19496 (2017).

[12] D. Benedikovic, et al., Photon. Res. 7, 437-444 (2019). 